Research Article

\title{
Distributions of the Ratio and Product of Two Independent Weibull and Lindley Random Variables
}

\author{
N. J. Hassan ${ }^{(D)}{ }^{1}$ A. Hawad Nasar, ${ }^{2}$ and J. Mahdi Hadad ${ }^{2}$ \\ ${ }^{1}$ Department of Mathematics, Faculty of Education for Pure Sciences, University of Thiqar, Al Nasiriyah, Iraq \\ ${ }^{2}$ Ministry of Education, Al Nasiriyah, Iraq \\ Correspondence should be addressed to N. J. Hassan; nabilhassan107@yahoo.com
}

Received 26 November 2019; Accepted 13 April 2020; Published 1 May 2020

Academic Editor: Alessandro De Gregorio

Copyright $\odot 2020$ N. J. Hassan et al. This is an open access article distributed under the Creative Commons Attribution License, which permits unrestricted use, distribution, and reproduction in any medium, provided the original work is properly cited.

In this paper, we derive the cumulative distribution functions (CDF) and probability density functions (PDF) of the ratio and product of two independent Weibull and Lindley random variables. The moment generating functions (MGF) and the $k$-moment are driven from the ratio and product cases. In these derivations, we use some special functions, for instance, generalized hypergeometric functions, confluent hypergeometric functions, and the parabolic cylinder functions. Finally, we draw the PDF and CDF in many values of the parameters.

\section{Introduction}

The distributions of ratio of random variables are widely used in many applied problems of engineering, physics, number theory, order statistics, economics, biology, genetics, medicine, hydrology, psychology, classification, and ranking and selection $[1,2]$. Examples include safety factor in engineering, mass to energy ratios in nuclear physics, target to control precipitation in meteorology, inventory ratios in economics, and Mendelian inheritance ratios in genetics [3, 4]. Also, ratio distribution involving two Gaussian random variables is used in computing error and outage probabilities [5]. It has many applications especially in engineering concepts such as structures, deterioration of rocket motors, static fatigue of ceramic components, fatigue failure of aircraft structures, and aging of concrete pressure vessels [6, 7]. An important example of ratios of random variables is the stress-strength model in the context of reliability. It describes the life of a component which has a random strength and is subjected to random stress. The general numerical method is developed for computing the PDF of the sums, products, or ratio of any number of nonnegative independent random variables [8]. The ratio and product distributions have been studied by several authors especially when independent random variables come from the same family or different families. For ratio distribution, the historical review, see $[9,10]$ for the normal family, [11] for Student's $t$ family, [12] for the Weibull family, [13] for the noncentral chi-squared family, [14] for the gamma family, [15] for the beta family, [16] for the logistic family, [17] for the Frechet family, [3] for the inverted gamma family, [18] for Laplace family, [7] for the generalized-F family, [19] for the hypoexponential family, [2] for the gamma and Rayleigh families, and [20] for gamma and exponential families. For product distribution, the historical review, see [21] for $t$ and Rayleigh families, [4] for Pareto and Kumaraswamy families, [6] for the $t$ and Bessel families, and [22] for the independent generalized gammaratio family. A new distribution is introduced based on compounding Weibull and Lindley distributions; in this approach, several properties of the distribution are derived [23]. In this paper, we derived the ratio and product of cumulative distribution functions (CDF) and probability density functions (PDF) of the independent Weibull and Lindley random variables. In this derivation, we used some special functions and integrals, for instance, generalized and 
confluent hypergeometric functions, parabolic cylinder function, gamma function of negative integer numbers, and some special integrals. We derived the moment generated function (MGF), m-moment, mean, and variance of the ratio random variable $Z=X / Y$, while we derived the $C D F, P D F$, and the MGF with respect to the product random variable $X Y$. The rest of this paper is organized as follows: in Section 2 , the derivation of the CDF, PDF, CGF, plots of the CDF and PDF, m-moment, mean, and variance of $Z=X / Y$ by using some special functions and integrals are given such that $X$ has Weibull distribution and $Y$ has Lindley distribution. The CDF, PDF, CGF, and plots of the CDF and PDF have been given in Section 3. Finally, some conclusions are considered in Section 4. Weibull distribution $X \sim W(a, b)$ with shape parameter $a>0$ and scale parameter $b>0$, the $\mathrm{PDF}$ and $\mathrm{CDF}$ are defined as

$$
f(x)=\frac{a}{b^{a}} x^{a-1} \exp \left[-\left(\frac{x}{b}\right) a\right] .
$$

And the CDF is

$$
F(x)=1-\exp \left[-\left(\frac{x}{b}\right) a\right]
$$

such that $X>0$. Lindley distribution $Y \sim L(c)$ with shape parameter $c>0$, the PDF and CDF are given as

$$
f(y)=\frac{c^{2}}{c+1}(y+1) \exp [-c y] .
$$

Also, the CDF is

$$
F(y)=1-\frac{(c+c y+1)}{c+1} \exp [-c y]
$$

such that $Y>0$. In this work, we assume that the shape parameter $a=2$ in Weibull distribution. So, the PDF, equation (1), and CDF, equation (2), become, respectively,

$$
\begin{aligned}
& f(x)=\frac{2}{b^{2}} x \exp \left[-\left(\frac{x}{b}\right)^{2}\right] . \\
& F(x)=1-\exp \left[-\left(\frac{x}{b}\right)^{2}\right] .
\end{aligned}
$$

The generalized hypergeometric function [24] is defined as

$$
{ }_{a} \Upsilon_{b}\left(d_{1}, d_{2}, \ldots, d p ; e_{1}, e_{2}, \ldots, e_{q} ; w\right)=\sum_{l=0}^{\infty}\left[\frac{\left(d_{1}\right)_{l}\left(d_{2}\right)_{2} \ldots\left(d_{p}\right)_{l} w^{l}}{\left(e_{1}\right)_{l}\left(e_{2}\right)_{2} \ldots\left(e_{q}\right)_{l} l !}\right],
$$

where Pochhammer symbol $(d)_{l}=(\Gamma(d+l)) / \Gamma(d)$ or $(e)_{l}=(\Gamma(e+l)) / \Gamma(e)$. The confluent hypergeometric function [24] is defined as

$$
\begin{aligned}
\Psi(d, e ; w)= & \frac{\Gamma(1-e)}{\Gamma(d-e+1)}\left[{ }_{1} \Upsilon_{1}(d ; e ; w)\right] \\
& +\frac{\Gamma(e-1)}{\Gamma(d)} w^{1-e}\left[{ }_{1} \Upsilon_{1}(d-e+1 ; 2-e ; w)\right],
\end{aligned}
$$

where $w \in \Re$ and $e \neq 0, \pm 1, \pm 2, \pm 3, \ldots$. The gamma function of the negative integer numbers [25] is given by

$$
\Gamma(-r)=\frac{(-1)^{r}}{r !} \phi(r)-\frac{(-1)^{r}}{r !} \eta,
$$

where $\phi(r)=\sum_{l=1}^{r} 1 / l$ and $\eta=\lim _{n \longrightarrow \infty} \sum_{l=1}^{n}((1) /(l-\ln (n)))$ is Euler's constant [25] such that $\Gamma(0)=-\eta$. The parabolic cylinder function [24] is defined as

$$
\Lambda_{v}(w)=2^{v / 2} \exp \left[-\frac{w^{2}}{4}\right] \Psi\left(-\frac{v}{2}, \frac{1}{2} ; \frac{w^{2}}{2}\right) .
$$

The first special integral [26] is given as

$$
\begin{aligned}
\int_{0}^{\infty} x^{\tau-1} \exp \left[-\rho x^{2}-\sigma x\right] d x= & (2 \rho)^{-(\tau / 2)} \Gamma(\tau) \exp \left[\left(\frac{\sigma^{2}}{8 \rho}\right)\right] \\
& \times \Lambda_{-\tau}\left(\frac{\sigma}{\sqrt{2 \rho}}\right),
\end{aligned}
$$

where $\operatorname{Re}(\sigma)>0$ and $\operatorname{Re}(\rho)>0$. The other special integral [26] is introduced:

$$
\int_{0}^{\infty} x^{\tau-1} \Psi(d, e ; x) \mathrm{d} x=\frac{\Gamma(\tau) \Gamma(d-\tau) \Gamma(\tau-e+1)}{\Gamma(d) \Gamma(d-e+1)} .
$$

The m-moment can be defined as

$$
E\left(Z^{m}\right)=\int_{0}^{\infty} Z^{m} f(z) \mathrm{d} z
$$

\section{Distribution of the Ratio $Z==X / Y$}

In this section, we derive CDF, PDF, MGF, plots of the CDF and PDF, m-moment, mean, and variance of $Z=X / Y$ by using some special functions. Let $X \sim W(2, b)$ and $Y \sim L(c)$; then, it can be found that the CDF of $Z=X / Y$ by

$$
F(z)=\operatorname{Pro}(Z \leq z)=\operatorname{Pro}\left(\frac{X}{Y} \leq z\right)=\int_{0}^{\infty} F_{X}(z y) f(y) \mathrm{d} y .
$$

By substituting equations (3) and (6) in equation (14), we get 


$$
\begin{aligned}
F(z)= & \int_{0}^{\infty}\left(1-\exp \left[-\frac{z^{2} y^{2}}{b^{2}}\right]\right) \frac{c^{2}}{c+1}(y+1) \exp [-c y] \\
= & \frac{c^{2}}{c+1} \int_{0}^{\infty}(1+y) \exp [-c y] \mathrm{d} y-\frac{c^{2}}{c+1} \int_{0}^{\infty}(1+y) \exp \left[-\frac{z^{2} y^{2}}{b^{2}}-c y\right] \mathrm{d} y \\
= & \frac{c^{2}}{c+1} \int_{0}^{\infty}(1+y) \exp [-c y] \mathrm{d} y-\frac{c^{2}}{c+1} \int_{0}^{\infty} y^{1-1} \exp \left[-\frac{z^{2} y^{2}}{b^{2}}-c y\right] \mathrm{d} y \\
& -\frac{c^{2}}{c+1} \int_{0}^{\infty} y^{2-1} \exp \left[-\frac{z^{2} y^{2}}{b^{2}}-c y\right] \mathrm{d} y .
\end{aligned}
$$

By using the first special integral in equation (11) to calculate the integral $\left(c^{2}\right) /(c+1) \int_{0}^{\infty} y^{1-1} \exp \left[-\left(z^{2} y^{2}\right) /\right.$

$\left.\left(b^{2}\right)-c y\right] \mathrm{d} y$ and the integral $\left(c^{2}\right) /(c+1) \int_{0}^{\infty} y^{2-1}$ $\exp \left[-\left(z^{2} y^{2}\right) /\left(b^{2}\right)-c y\right] \mathrm{d} y$, one can obtain

$$
\begin{aligned}
\frac{c^{2}}{c+1} \int_{0}^{\infty} y^{1-1} \exp \left[-\frac{z^{2} y^{2}}{b^{2}}-c y\right] \mathrm{d} y & =\left(\frac{2 z^{2}}{b^{2}}\right)^{-(1 / 2)} \exp \left[\frac{b^{2} c^{2}}{8 z^{2}}\right] \Lambda_{-1}\left(\frac{b c}{\sqrt{2} z}\right) \\
& =\frac{b}{2 z} \Psi\left(\frac{1}{2}, \frac{1}{2} ; \frac{b^{2} c^{2}}{4 z^{2}}\right), \\
\frac{c^{2}}{c+1} \int_{0}^{\infty} y^{2-1} \exp \left[-\frac{z^{2} y^{2}}{b^{2}}-c y\right] \mathrm{d} y & =\left(\frac{2 z^{2}}{b^{2}}\right)^{-1} \exp \left[\frac{b^{2} c^{2}}{8 z^{2}}\right] \Lambda_{-2}\left(\frac{b c}{\sqrt{2} z}\right) \\
& =\frac{b^{2}}{4 z^{2}} \Psi\left(1, \frac{1}{2} ; \frac{b^{2} c^{2}}{4 z^{2}}\right) .
\end{aligned}
$$

We substitute equations (16) and (17) in equation (15):

$$
\begin{aligned}
F(z)= & 1-\frac{c^{2}}{c+1} \frac{b}{2 z} \Psi\left(\frac{1}{2}, \frac{1}{2} ; \frac{b^{2} c^{2}}{4 z^{2}}\right) \\
& -\frac{b^{2}}{4 z^{2}} \Psi\left(1, \frac{1}{2} ; \frac{b^{2} c^{2}}{4 z^{2}}\right) .
\end{aligned}
$$

Equation (18) represents the CDF of $Z=X / Y$, where $X \sim W(2, b)$ and $Y \sim L(c)$ by the confluent hypergeometric function $\Psi(d, e ; w)$. We can analysis equation (18) as the following picture:

$$
\begin{aligned}
F(z)= & 1-\frac{c^{2}}{c+1} \frac{b}{2 z} \sqrt{\pi}\left[\Upsilon_{1}\left(0.5 ; 0.5 ; \frac{b^{2} c^{2}}{4 z^{2}}\right)\right] \\
& -\left(\frac{b c}{2 z}\right)\left[{ }_{1} \Upsilon_{1}\left(1 ; \frac{3}{2} ; \frac{b^{2} c^{2}}{4 z^{2}}\right)\right] \\
& -\frac{b^{2}}{2 z^{2}}\left[{ }_{1} \Upsilon_{1}\left(1 ; 0.5 ; \frac{b^{2} c^{2}}{4 z^{2}}\right)\right] \\
& -\sqrt{\pi}\left(\frac{b c}{z}\right)\left[{ }_{1} \Upsilon_{1}\left(\frac{3}{2} ; \frac{3}{2} ; \frac{b^{2} c^{2}}{4 z^{2}}\right)\right] .
\end{aligned}
$$

Equation (19) is CDF of $Z$ by the generalized hypergeometric function ${ }_{p} \Upsilon_{q}\left(d_{1}, d_{2}, \ldots, d_{p} ; e_{1}, e_{2}, \ldots, e_{q} ; w\right)$.
The PDF of $Z=X / Y$ such that $X \sim W(2, b)$ and $Y \sim L(c)$ can be defined as

$$
f(z)=\operatorname{Pro}(Z=z)=\operatorname{Pro}\left(\frac{X}{Y}=z\right)=\int_{0}^{\infty} y f_{X}(z y) f(y) \mathrm{d} y .
$$

By substituting equations (3) and (5), one can obtain

$$
\begin{aligned}
f(z)= & \operatorname{Pro}(Z=z)=\int_{0}^{\infty} \frac{2}{b^{2}} z y^{2} \exp \left[-\left(\frac{z y}{b}\right)^{2}\right] \\
& \frac{c^{2}}{c+1}(y+1) \exp (-c y) \mathrm{d} y, \\
f(z)= & \frac{2 z c^{2}}{b^{2}(c+1)} \int_{0}^{\infty}\left(y^{3}+y^{2}\right) \exp \left[-\frac{z^{2} y^{2}}{b^{2}}-c y\right] \mathrm{d} y \\
= & \frac{2 z c^{2}}{b^{2}(c+1)}\left[\int_{0}^{\infty} y^{3-1} \exp \left[-\frac{z^{2} y^{2}}{b^{2}}-c y\right] \mathrm{d} y\right. \\
& \left.+\int_{0}^{\infty} y^{4-1} \exp \left[-\frac{z^{2} y^{2}}{b^{2}}-c y\right] \mathrm{d} y\right] .
\end{aligned}
$$

By using the first special integral in equation (11) to compute the integrals $\int_{0}^{\infty} y^{3-1} \exp \left[-\left(\left(z^{2} y^{2}\right) /\left(b^{2}\right)\right)-c y\right] \mathrm{d} y$ and $\int_{0}^{\infty} y^{4-1} \exp \left[-\left(\left(z^{2} y^{2}\right) /\left(b^{2}\right)\right)-c y\right] \mathrm{d} y$, one can obtain 
$f(z)=\frac{2 z c^{2}}{b^{2}(c+1)}\left[2^{-2} \frac{b^{3}}{z^{3}} \Psi\left(\frac{3}{2}, \frac{1}{2} ; \frac{b^{2} c^{2}}{4 z^{2}}\right)+\frac{3 b^{4}}{8 z^{4}} \Psi\left(2, \frac{1}{2} ; \frac{b^{2} c^{2}}{4 z^{2}}\right)\right]$.

Equation (23) is PDF of $Z=X / Y$, where $X \sim W(2, b)$ and $Y \sim L(c)$ by the confluent hypergeometric function. Figure 1 is the plot of CDF in equation (18).

Figure 2 is the plot of PDF in equation (23).

By substituting equation (8) in equation (23), we have

$$
\begin{aligned}
f(z)= & \frac{2 z c^{2}}{b^{2}(c+1)} 2^{-2} \frac{b^{3}}{z^{3}} \sqrt{\pi}\left[\Upsilon_{1} \Upsilon_{1}\left(\frac{3}{2} ; \frac{1}{2} ; \frac{b^{2} c^{2}}{4 z^{2}}\right)\right] \\
& -4 \frac{c b}{\sqrt{2} z}\left[{ }_{1} \Upsilon_{1}\left(2 ; \frac{3}{2} ; \frac{b^{2} c^{2}}{4 z^{2}}\right)\right] \\
& +\frac{2 z c^{2}}{b^{2}(c+1)} \frac{3 b^{4}}{8 z^{4}} \frac{4}{3}\left[{ }_{1} \Upsilon_{1}\left(2 ; \frac{1}{2} ; \frac{b^{2} c^{2}}{4 z^{2}}\right)\right] \\
& -2 \sqrt{\pi} \frac{c b}{\sqrt{2} z}\left[{ }_{1} \Upsilon_{1}\left(\frac{5}{2} ; \frac{1}{2} ; \frac{b^{2} c^{2}}{4 z^{2}}\right)\right] .
\end{aligned}
$$

Equation (24) is PDF of $z$-generalized hypergeometric function. We put equation (23) in equation (13) to get the following:

$$
\begin{aligned}
E\left(Z^{m}\right)= & \frac{2}{b(c+1)}\left[\int_{0}^{\infty} Z^{m} \frac{b^{2} c^{2}}{4 z^{2}} \Psi\left(\frac{3}{2}, \frac{1}{2} ; \frac{b^{2} c^{2}}{4 z^{2}}\right) \mathrm{d} z\right] \\
& +\frac{3}{c+1}\left[\int_{0}^{\infty} Z^{m-1} \frac{b^{2} c^{2}}{4 z^{2}} \Psi\left(2, \frac{1}{2} ; \frac{b^{2} c^{2}}{4 z^{2}}\right) \mathrm{d} z\right] \\
= & \frac{-(c b / 2)^{m+1}}{b(c+1)}\left[\int_{0}^{\infty} \omega^{((1-m) / 2)-1} \Psi\left(\frac{3}{2}, \frac{1}{2} ; \omega\right) \mathrm{d} \omega\right] \\
& -\frac{3}{2(c+1)}\left(\frac{c b}{2}\right)^{m}\left[\int_{0}^{\infty} \omega^{(2-m / 2)-1} \Psi\left(2, \frac{1}{2} ; \frac{b^{2} c^{2}}{4 z^{2}}\right) \mathrm{d} \omega\right]
\end{aligned}
$$

By using the second special integral in equation (12), we obtain as

$$
\begin{aligned}
E\left(Z^{m}\right)= & \frac{(c b / 2)^{m+1}}{b(c+1)}\left[\frac{\Gamma(1-m / 2) \Gamma(2+m / 2) m \Gamma(-m / 2)}{\sqrt{\pi}}\right] \\
& -\frac{3}{2(c+1)}\left(\frac{c b}{2}\right)^{m}\left[4 \frac{\Gamma(2-m / 2) \Gamma(2+m / 2) \Gamma(3-m / 2)}{3 \sqrt{\pi}}\right]
\end{aligned}
$$

Equation (27) is m-moment of $Z$.

Now, if $m=1$, the mean of $Z$ in equation (27) is

$$
E(Z)=\frac{(c b / 2)^{2}}{b(c+1)}[-\sqrt{\pi} \eta]-\frac{3}{2(c+1)}\left(\frac{c b}{2}\right)\left[\frac{2 \sqrt{\pi}}{3}\right] .
$$

If $m=2$, the second moment in equation (27) is

$$
E\left(Z^{2}\right)=\frac{(c b / 2)^{3}}{b(c+1)} 4[1-\eta]-\frac{3}{2(c+1)}\left(\frac{c b}{2}\right)^{2}\left[\frac{-4 \eta}{3}\right] .
$$

The variance $V(Z)$ can be found from equations (28) and (29) as

$$
\begin{aligned}
V(Z)= & \frac{(c b / 2)^{3}}{b(c+1)} 4[1-\eta]-\frac{3}{2(c+1)}\left(\frac{c b}{2}\right)^{2}\left[\frac{-4 \eta}{3}\right] \\
& -\left[\frac{(c b / 2)^{2}}{b(c+1)}[-\sqrt{\pi} \eta]-\frac{3}{2(c+1)}\left(\frac{c b}{2}\right)\left[\frac{2 \sqrt{\pi}}{3}\right]\right]^{2}
\end{aligned}
$$

The MGF of $Z$ can be calculated as

$$
\begin{aligned}
M_{Z}(t)= & E(\exp [t z]) \\
= & \frac{2}{b(c+1)}\left[\int_{0}^{\infty} \exp [t z] \frac{b^{2} c^{2}}{4 z^{2}} \Psi\left(\frac{3}{2}, \frac{1}{2} ; \frac{b^{2} c^{2}}{4 z^{2}}\right) \mathrm{d} z\right] \\
& +\frac{3}{c+1}\left[\int_{0}^{\infty} z^{-1} \exp [t z] \frac{b^{2} c^{2}}{4 z^{2}} \Psi\left(2, \frac{1}{2} ; \frac{b^{2} c^{2}}{4 z^{2}}\right) \mathrm{d} z\right] \\
= & -\frac{c}{2(c+1)}\left[\int_{0}^{\infty} \omega^{-1 / 2} \exp \left[\frac{c b \omega^{-1 / 2} t}{2}\right] \Psi\left(\frac{3}{2}, \frac{1}{2} ; \omega\right) \mathrm{d} \omega\right] \\
& -\frac{3}{2(c+1)}\left[\int_{0}^{\infty} \exp \left[\frac{c b \omega^{-1 / 2} t}{2}\right] \Psi\left(2, \frac{1}{2} ; \omega\right) \mathrm{d} \omega\right],
\end{aligned}
$$

where $\omega=\left(b^{2} c^{2}\right) /\left(4 z^{2}\right)$. Using equations (7) and (8) to rewrite equation (31) as the following picture,

$$
\begin{aligned}
M_{Z}(t)= & {\left[\frac{2 c}{(c+1)} \sum_{l=0}^{\infty} \frac{(2)_{l}}{(3 / 2)_{l} l !}-\frac{2}{c+1} \sum_{l=0}^{\infty} \frac{(2)_{l}}{(1 / 2)_{l} l !}\right] } \\
& \times \int_{0}^{\infty} \omega^{l} \exp \left[\frac{c b \omega^{-1 / 2} t}{2}\right] \mathrm{d} \omega+\left[-\frac{\sqrt{\pi} c}{2(c+1)} \sum_{l=0}^{\infty} \frac{(3 / 2)_{l}}{(1 / 2)_{l} l !}\right] \\
& \times \int_{0}^{\infty} \omega^{l-(1 / 2)} \exp \left[\frac{c b \omega^{-1 / 2} t}{2}\right] \mathrm{d} \omega \\
& +\left[\frac{3 \sqrt{\pi}}{c+1} \sum_{l=0}^{\infty} \frac{(5 / 2)_{l}}{(3 / 2)_{l} l !}\right] \int_{0}^{\infty} \omega^{l+(1 / 2)} \exp \left[\frac{c b \omega^{-1 / 2} t}{2}\right] \mathrm{d} \omega .
\end{aligned}
$$

To solve the integrals in equation (32), we assume that $-R=\left(c b \omega^{(-1) / 2} t\right) /(2)$; then, equation (32) becomes

$$
\begin{aligned}
M_{Z}(t)= & -\left[\frac{2 c}{(c+1)} \sum_{l=0}^{\infty} \frac{(2)_{l}}{(3 / 2)_{l} l !}-\frac{2}{c+1} \sum_{l=0}^{\infty} \frac{(2)_{l}}{(1 / 2)_{l} l !}\right] \\
& \times \frac{(b c t)^{2 l} \Gamma(-(2 l+2))}{2^{2 l+1}}+\left[-\frac{\sqrt{\pi} c}{2(c+1)} \sum_{l=0}^{\infty} \frac{(3 / 2)_{l}}{(1 / 2)_{l} l !}\right] \\
& \times(-2)\left(\frac{b c t}{2}\right)^{2 l+1} \Gamma(-(2 l+1))+\left[\frac{3 \sqrt{\pi}}{c+1} \sum_{l=0}^{\infty} \frac{(5 / 2)_{l}}{(3 / 2)_{l} l !}\right] \\
& \times(-2)\left(\frac{b c t}{2}\right)^{2 l+3} \Gamma(-(2 l+3)) .
\end{aligned}
$$




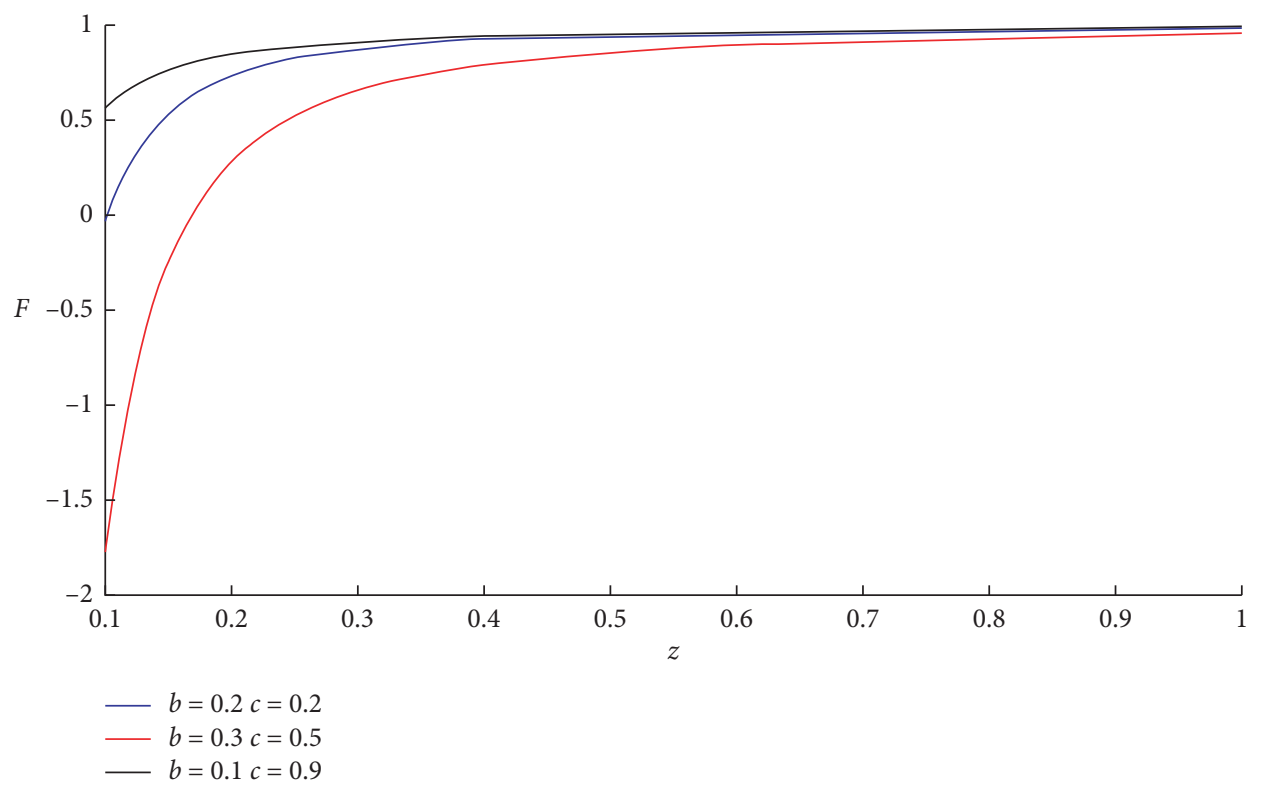

FIGURE 1: The graph of CDF for equation (18); we take three cases of the values of the parameters: first case $b=c=0.2$, second case $b=0.3$ and $c=0.5$, and final case $b=0.1$ and $c=0.9$.

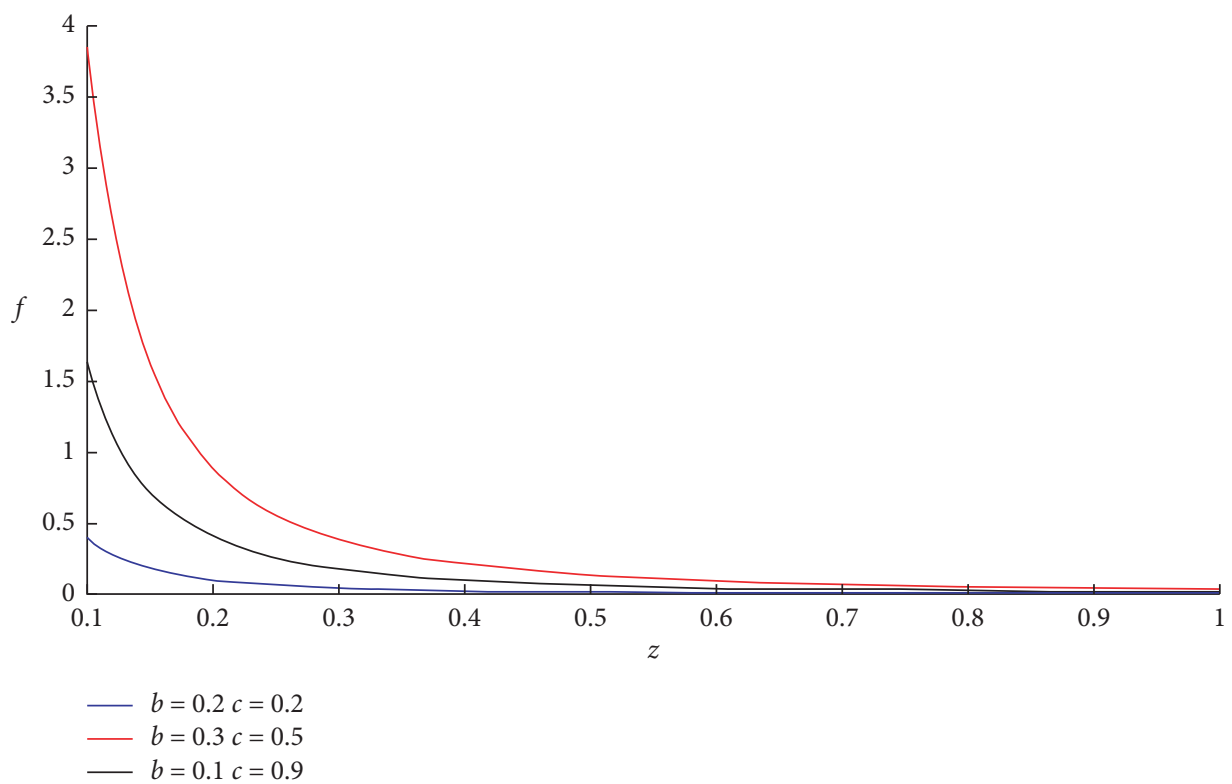

Figure 2: The graph of PDF for equation (23); we take three cases of the values of the parameters: first case $b=c=0.2$, second case $b=0.3$ and $c=0.5$, and final case $b=0.1$ and $c=0.9$. 
where $\Gamma(-(2 l+2)), \Gamma(-(2 l+1))$, and $\Gamma(-(2 l+3))$ are gamma functions of the negative integer numbers.

\section{Distribution of the Product $Z==X Y$}

In this section, we derive CDF, PDF, MGF, plots of the CDF and PDF, m-moment, mean, and variance of $Z=X Y$ by using some special functions. Let $X \sim W(2, b)$ and $Y \sim L(c)$; then, the CDF of $Z=X Y$ can be computed by

$$
\begin{aligned}
F(z) & =\operatorname{Pro}\left(X \leq \frac{y}{z}\right)=\int_{0}^{\infty} F_{X}\left(\frac{y}{z}\right) f(y) \mathrm{d} y \\
& =\int_{0}^{\infty}\left[1-\exp \left[\frac{-y^{2}}{b^{2} z^{2}}\right]\right] \frac{c^{2}}{c+1}(y+1) \exp [-c y] \mathrm{d} y \\
& =1-\frac{c^{2}}{c+1} \frac{b z}{2} \Psi\left(\frac{1}{2}, \frac{1}{2} ; \frac{b^{2} c^{2} z^{2}}{4}\right)-\frac{c^{2}}{c+1} \frac{b^{2} c^{2}}{4} \Psi\left(1, \frac{1}{2} ; \frac{b^{2} c^{2} z^{2}}{4}\right) .
\end{aligned}
$$

Equation (33) is CDF of $Z=X Y$ by the confluent hypergeometric function. By using equation (8), equation (33) can be written as the following:

$$
\begin{aligned}
F(z)= & 1-\frac{b z c^{2}}{2(c+1)}\left[\sqrt{\pi}\left[{ }_{1} \Upsilon_{1}\left(\frac{1}{2} ; \frac{1}{2} ; \theta\right)\right]-2 \theta^{1 / 2}\left[{ }_{1} \Upsilon_{1}\left(\frac{1}{2} ; \frac{1}{2} ; \theta\right)\right]\right] \\
& -\frac{b^{2} c^{4}}{4(c+1)}\left[2\left[{ }_{1} \Upsilon_{1}\left(1 ; \frac{1}{2} ; \theta\right)\right]-2 \sqrt{\pi} \theta^{1 / 2}\left[{ }_{1} \Upsilon_{1}\left(\frac{3}{2} ; \frac{3}{2} ; \theta\right)\right]\right]
\end{aligned}
$$

$$
\begin{aligned}
& f(z)=\int_{0}^{\infty} \frac{1}{x} f_{Y}\left(\frac{z}{x}\right) f_{X}(x) \mathrm{d} x \\
& =\frac{2 c^{2}}{b^{2}(c+1)} \int_{0}^{\infty}\left(1+\frac{z}{x}\right) \exp \left[-\frac{x^{2}}{b^{2}}\right] \exp \left[-\frac{c z}{x}\right] \mathrm{d} x \\
& =\frac{2 c^{2}}{b^{2}(c+1)} \int_{0}^{\infty}\left(1+\frac{z}{x}\right) \exp \left[-\frac{x^{2}}{b^{2}}\right]\left[\sum_{l=0}^{\infty} \frac{(-1)^{l}(c z)^{l} x^{-l}}{k !}\right] \\
& =\frac{2 c^{2}}{b^{2}(c+1)} \sum_{l=0}^{\infty} \frac{(-1)^{l}(c z)^{l}}{l !}\left[\int_{0}^{\infty} x^{-l} \exp \left[-\left[\frac{x^{2}}{b^{2}}\right]\right]\left[+z \int_{0}^{\infty} x^{-l-1} \exp \left[-\left(\frac{x^{2}}{b^{2}}\right)\right] \mathrm{d} x\right]\right. \\
& =\frac{2 c^{2}}{b^{2}(c+1)} \sum_{l=0}^{\infty} \frac{(-1)^{l}(c z)^{l}}{l !}\left[\frac{b^{-l+1}}{2} \Gamma\left(\frac{-l+1}{2}\right)\left[+z \frac{b^{-l}}{2} \Gamma\left(\frac{-l}{2}\right)\right],\right.
\end{aligned}
$$

where $R=\left(x^{2}\right) /\left(b^{2}\right)$. So,

$$
f(z)=\frac{2 c^{2}}{b^{2}(c+1)} \sum_{l=0}^{\infty} \frac{(-1)^{l}(c z)^{l}}{l !}\left[\frac{b^{-l+1}}{2} \Gamma\left(\frac{-l+1}{2}\right)+z \frac{b^{-l}}{2} \Gamma\left(\frac{-l}{2}\right)\right] .
$$

Equation (34) is CDF of $Z$ by generalized hypergeometric function, where $\theta=\left(b^{2} c^{2} z^{2}\right) /(4)$. The PDF of $Z=$ $X Y$ can be considered: 


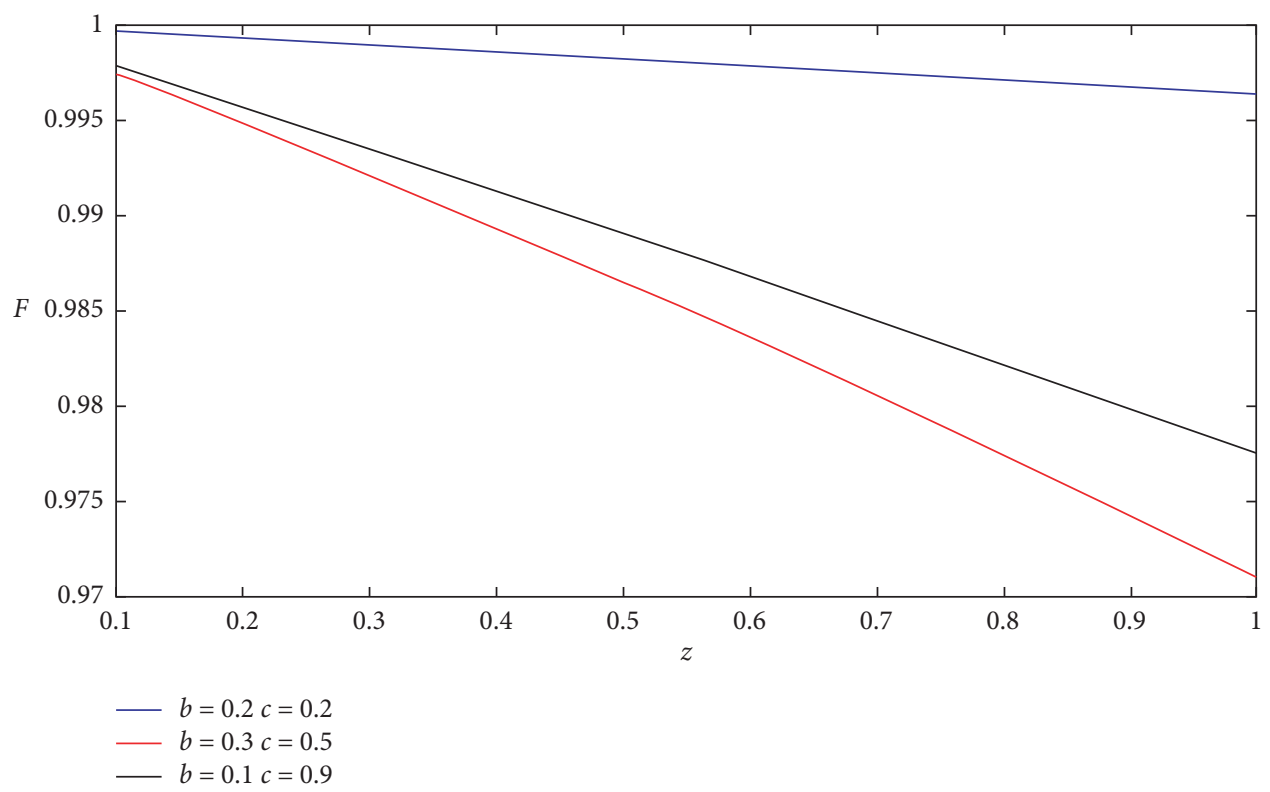

Figure 3: The graph of CDF for equation (33); we take three cases of the values of the parameters: first case $b=c=0.2$, second case $b=0.3$ and $c=0.5$, and final case $b=0.1$ and $c=0.9$.

$$
\begin{aligned}
M_{Z}(t)= & \frac{2 c^{2}}{b^{2}(c+1)} \sum_{l=0}^{\infty} \frac{(-1)^{l}(c)^{l}}{l !}\left[\frac{b^{-l+1}}{2} \Gamma\left(\frac{-l+1}{2}\right) \int_{0}^{\infty} z^{l} \exp [t z]\right. \\
& \left.+\frac{b^{-l}}{2} \Gamma\left(\frac{-l}{2}\right) \int_{0}^{\infty} z^{l+1} \exp [t z]\right] \\
= & \frac{2 c^{2}}{b^{2}(c+1)} \sum_{l=0}^{\infty} \frac{(-1)^{l}(c)^{l}}{l !}\left[\frac{b^{-l+1}}{2} \Gamma\left(\frac{-l+1}{2}\right)\left[\frac{(-1)^{l} \Gamma(l+1)}{t^{l+1}}\right]\right. \\
& \left.+\frac{b^{-l}}{2} \Gamma\left(\frac{-l}{2}\right)\left[\frac{(-1)^{l} \Gamma(l+2)}{t^{l+2}}\right]\right]
\end{aligned}
$$

where $-R=t z$. Therefore,

$$
\begin{aligned}
M_{Z}(t)= & \frac{2 c^{2}}{b^{2}(c+1)} \sum_{l=0}^{\infty} \frac{(-1)^{l}(c)^{l}}{l !}\left[\frac{b^{-l+1}}{2} \Gamma\left(\frac{-l+1}{2}\right)\left[\frac{(-1)^{l} \Gamma(l+1)}{t^{l+2}}\right]\right. \\
& +\left[\frac{b^{-l}}{2} \Gamma\left(\frac{-l}{2}\right)\left[\frac{(-1)^{l} \Gamma(l+2)}{t^{l+2}}\right]\right] .
\end{aligned}
$$

Equation (38) is MGF of $Z$.

\section{Conclusion}

In this paper, we discussed distribution of the ratio independent Weibull and Lindley random variables. In this approach, we derived the CDF, PDF, and MGF of the ratio independent Weibull and Lindley random variables. Also, the plots of CDF and PDF are drawn. The m-moment, mean, and variance are calculated. The CDF and PDF are derived in two formulas with respect to each one of them, first formula by confluent hypergeometric function and another formula by generalized hypergeometric function.
We studied distribution of the product independent Weibull and Lindley random variables. In this case, the CDF, PDF, and MGF are derived from the product independent Weibull and Lindley random variables. The plots of CDF and PDF are drawn. The CDF is derived in two formulas: first formula by using confluent hypergeometric function and another formula by using generalized hypergeometric function. However, PDF is derived by using gamma function of negative integer numbers.

\section{Data Availability}

The data used to support the findings of this study are included within the article.

\section{Conflicts of Interest}

The authors declare that they have no conflicts of interest.

\section{Acknowledgments}

The authors thank every person who helped them to complete this paper.

\section{References}

[1] S. Nadarajah and D. Choi, "Arnold and Strauss's bivariate exponential distribution products and ratios," New Zealand Journal of Mathematics, vol. 35, pp. 189-199, 2006.

[2] M. Shakil and B. M. G. Kibria, "Exact distribution of the ratio of gamma and Rayleigh random variables," Pakistan Journal of Statistics and Operation Research, vol. 2, no. 2, pp. 87-98, 2006.

[3] M. M. Ali, M. Pal, and J. Woo, "On the ratio of inverted gamma variates," Austrian Journal of Statistic, vol. 36, no. 2, pp. 153-159, 2007. 
[4] L. Idrizi, "On the product and ratio of Pareto and Kumaraswamy random variables," Mathematical Theory and Modeling, vol. 4, no. 3, pp. 136-146, 2014.

[5] S. Park, "On the distribution functions of ratios involving Gaussian random variables," ETRI Journal, vol. 32, no. 6, 2010.

[6] S. Nadarajah and S. Kotz, "On the product and ratio of $\mathrm{t}$ and Bessel random variables," Bulletin of the Institute of Mathematics Academia Sinica, vol. 2, no. 1, pp. 55-66, 2007.

[7] T. Pham-Gia and N. Turkkan, "Operations on the generalized-fvariables and applications," Statistics, vol. 36, no. 3, pp. 195-209, 2002.

[8] G. Beylkin, L. Monzón, and I. Satkauskas, "On computing distributions of products of non-negative independent random variables," Applied and Computational Harmonic Analysis, vol. 46, no. 2, pp. 400-416, 2019.

[9] P. J. Korhonen and S. C. Narula, "The probability distribution of the ratio of the absolute values of two normal variables," Journal of Statistical Computation and Simulation, vol. 33, no. 3, pp. 173-182, 1989.

[10] G. Marsaglia, "Ratios of normal variables and ratios of sums of uniform variables," Journal of the American Statistical Association, vol. 60, no. 309, pp. 193-204, 1965.

[11] S. J. Press, "Thet-ratio distribution," Journal of the American Statistical Association, vol. 64, no. 325, pp. 242-252, 1969.

[12] A. P. Basu and R. H. Lochner, "On the distribution of the ratio of two random variables having generalized life distributions," Technometrics, vol. 13, no. 2, pp. 281-287, 1971.

[13] D. L. Hawkins and C.-P. Han, "Bivariate distributions of some ratios of independent noncentral chi-square random variables," Communications in Statistics - Theory and Methods, vol. 15, no. 1, pp. 261-277, 1986.

[14] S. B. Provost, "On the distribution of the ratio of powers of sums of gamma random variables," Pakistan Journal Statistic, vol. 5, no. 2, pp. 157-174, 1989.

[15] T. Pham-Gia, "Distributions of the ratios of independent beta variables and applications," Communications in StatisticsTheory and Methods, vol. 29, no. 12, pp. 2693-2715, 2000.

[16] S. Nadarajah and A. K. Gupta, "On the ratio of logistic random variables," Computational Statistics \& Data Analysis, vol. 50, no. 5, pp. 1206-1219, 2006.

[17] S. Nadarajah and S. Kotz, "On the ratio of fréchet random variables,” Quality \& Quantity, vol. 40, no. 5, pp. 861-868, 2006.

[18] S. Nadarajah, "The linear combination, product and ratio of Laplace random variables," Statistics, vol. 41, no. 6, pp. 535-545, 2007.

[19] K. Therrar and S. Khaled, "The exact distribution of the ratio of two independent hypoexponential random variables," British Journal of Mathematics and Computer Science, vol. 4, no. 18, pp. 2665-2675, 2014.

[20] L. joshi and K. Modi, "On the distribution of ratio of gamma and three parameter exponentiated exponential random variables," Indian Journal of Statistics and Application, vol. 3, no. 12, pp. 772-783, 2014.

[21] K. Modi and L. Joshi, "On the distribution of product and ratio of $\mathrm{t}$ and Rayleigh random variables," Journal of the Calcutta Mathematical Society, vol. 8, no. 1, pp. 53-60, 2012.

[22] C. A. Coelho and J. T. Mexia, "On the distribution of the product and ratio of independent generalized gamma-ratio," Sankhya: The Indian Journal of Statistics, vol. 69, no. 2, pp. 221-255, 2007.
[23] A. Asgharzadeh, S. Nadarajah, and F. Sharafi, "Weibull lindley distributions," Statistical Journal, vol. 16, no. 1, pp. 87-113, 2018.

[24] A. P. Prudnikov, Y. A. Brychkov, and O. I. Marichev, Integrals and Series, Gordon and Breach Science Publishers, vol. 2, no. 3, Amsterdam, Netherlands, 1986.

[25] F. Brian and K. Adem, "Some results on the gamma function for negative integers," Applied Mathematics \& Information Sciences, vol. 6, no. 2, pp. 173-176, 2012.

[26] I. S. Gradshteyn and I. M. Ryzhik, Table of Integrals, Series and Products, vol. 6, Academic Press, Cambridge, MA, USA. 